\title{
The antioxidant effect of Echinacea angustifolia and Echinacea purpurea in rat colitis model induced by acetic acid
}

\author{
Dogan $\mathrm{Z}^{1}$, Ergul $\mathrm{B}^{1}$, Sarikaya $\mathrm{M}^{1}$, Filik $\mathrm{L}^{1}$, Alparslan Gonultas $\mathrm{M}^{2}$, Hucumenoglu $\mathrm{S}^{1}$, Can $\mathrm{M}^{3}$ \\ Ankara Education and Research Hospital, Gastroenterology Department, Sukriye District, Altindag, Ankara, \\ Turkey.doganzeynal@yahoo.com
}

\begin{abstract}
Background: Ulcerative colitis is a chronic inflammatory condition of the colon, and reactive oxidative metabolites (ROMs) play an important role in its pathogenesis. Alternative therapies such as herbal remedies are increasingly being used in the treatment of ulcerative colitis for better clinical outcome of ulcerative colitis and less adverse effects. Echinacea has many features including antioxidant and wound-healing properties. Hence, the present study was undertaken to evaluate the protective effect of Echinacea spp. on experimental colitis model induced by acetic acid in Wistar albino rats.

Methods: Acute colitis was induced by intrarectal administration of acetic acid. Rats were divided into four groups, namely control, Echinacea-administered, Echinacea-administered-colitis and colitis. Malondialdehyde and total antioxidant status were assayed in tissue samples. Histopathological evaluation was also performed. Results: Macroscopic and microscopic scores were significantly higher in colitis group compared to control, Echinacea and Echinacea-colitis groups $(p<0.001)$. There was no significant differences in respect of macroscopic and microscopic scores between control, Echinacea and Echinacea-colitis groups $(p>0.3, p>0.22)$. Malondialdehyde levels were elevated in colitis group compared to other groups $(p<0.001)$. Total antioxidant status was significantly higher in Echinacea group compared with other groups and also significantly higher in Echinacea-colitis group compared with colitis group ( $p<0.001, p<0.001$, respectively).

Conclusion: Echinacea may possibly have some therapeutic usefulness in the management of ulcerative colitis (Tab. 2, Fig. 4, Ref. 35). Text in PDF www.elis.sk.

Key words: Echinacea spp., ulcerative colitis, reactive oxygen metabolites.
\end{abstract}

Ulcerative colitis (UC) is a chronic inflammatory bowel disease of unknown origin. Current literature suggests that multiple genetic, environmental and immune factors take place in the pathogenesis and progression of the disease $(1,2,3,4)$. It has been well known for a long time that inflammatory mediators such as cytokines, eicosanoids and reactive oxygen metabolites (ROMs) are crucial components of those inflammatory pathways. These molecules are known to play a role in both the initiation and the progression of UC $(5,6)$. The production of these metabolites which are shown to be the final common mediators of inflammation has been found to occur in the gastrointestinal tract (7). In this regard, it is required to take antioxidants and dietary supplements that may affect the body's defence system against oxidants like hydrogen peroxide and others.

Echinacea spp., one of the oldest and most popular species in the world belonging to the family Asteraceae, is found abundantly throughout the world. Echinacea has many features including antiviral, antibacterial, antifungal, antioxidant, anticarcinogenic, antiinflammatory and wound-healing properties. A recent case-

${ }^{1}$ Ankara Education and Research Hospital, Gastroenterology, ${ }^{2}$ Ankara Education and Research Hospital, Pathology, ${ }^{3}$ Zonguldak Karaelmas University Medical School, Medical Biochemistry, Altindag, Ankara, Turkey

Address for correspondence: Z. Dogan, Ankara Education and Research Hospital, Gastroenterology Department, Sukriye District, Ulucanlar Street, 06340 Postal Code, Altindag, Ankara, Turkey.

Phone: +90.312 .5954273 , Fax: +90.312 .3633396$ control study shows the therapeutic role of Echinacea in children with mucositis caused by chemotherapy (8). There are nine known species of Echinacea. Therapeutic effects have been reported in three species, E. purpurea, E. pallida, and E. angustifolia, which are all used as medical herbs (9). Among their components, echinacin, a peculiar glucose chain derived from $E$. purpurea, has been reported to attach to the surface of T cells and macrophages and to activate them (10). Therefore, Echinacea may be used in UC due to its antioxidant effects.

Malondialdedyde (MDA) is a valid final product of lipid peroxidation by ROMs. MDA is also known as a thiobarbituric acid-reactive breakdown product which is assayed as an indirect marker of lipid peroxidation $(11,12)$. The level of MDA has been shown to be significantly higher in colitis models in previous studies $(13,14)$. TAS Assay measures the total antioxidant capacity of biomolecules from a variety of samples via a set mechanism. In the presence of antioxidants, copper (II) is reduced to copper (I). In turn, the copper (I) ions react with chromogen to produce a color with maximum absorbance at $490 \mathrm{~nm}$. It is suitable for being used with plasma, urine, cell lysates, tissue homogenates and food extracts. It works with a wide variety of antioxidants. ROMs are potent inflammatory mediators likely to be involved in tissue injury in colitis. These mediators may play an important role in the course of UC and its acute attacks. ROMs in early stage of colitis may increase and be a vital factor in the etiopathogenesis of colitis (15). It has been reported that antioxidants decrease the 
411-415

detrimental effects of oxidative damage caused by ROMs, and increase TAS in colitis models $(16,17)$

Drugs used in UC are not always effective and may cause serious side effects $(18,19)$. Therefore, new therapeutic strategies are awaited. That is why alternative therapies such as antioxidant agents and herbs are increasingly being used for the treatment of UC. Hence, the present study was undertaken to evaluate the protective and antioxidant effect of Echinacea spp. on experimental model of colitis induced by acetic acid in Wistar albino rats.

\section{Materials and methods}

Echinacea extract (Echinacea angustifolia and Echinacea purpurea) was provided in form of dried powder commercial preparation by GNC (General Nutrition Center), Pittsburgh, USA.

\section{Animals}

Twenty male Wistar Albino rats, weighing 200-260 g each, were obtained from Ankara Education and Research Hospital of Experimental Research Laboratory (Ankara, Turkey). They were kept in rat cages in a standard animal room, which was maintained under a $12 \mathrm{~h}$ light $/ 12 \mathrm{~h}$ dark cycle at $21-22^{\circ} \mathrm{C}$. Rats were allowed ad libitum access to food and water. The structure of this study and animal experimental procedures were approved by the Ethical Committee of Ankara Education and Research Hospital.

\section{Acute colitis induction and treatment protocols}

Acute colitis was induced by intrarectal administration of $1 \mathrm{ml}$ of a $4 \%$ solution of acetic acid. After ketamin anesthesia, a soft $6 \mathrm{~F}$ pediatric catheter was introduced $6 \mathrm{~cm}$ into the anus and acetic acid was carefully administered. Before taking the catheter out, $2 \mathrm{ml}$ of air was applied in order to spread acetic acid completely in the colon. All rats were maintained in head-down position for 25 seconds to prevent leakage. Echinacea $50 \mathrm{mg} / \mathrm{kg} /$ day (equal to human dosage) was given into the stomach by a soft $6 \mathrm{~F}$ pediatric catheter. Rats were randomized to four groups, five animals per group as follows:

Control (Group 1; $\mathrm{n}=5$ ) were allowed ad libitum access to food and water for 14 days. Echinacea (Group 2; $n=5$ ) were allowed ad libitum access to food, water and $50 \mathrm{mg} / \mathrm{kg}$ /day Echinacea for 14 days. On day eight, $2 \mathrm{ml}$ saline was administered into the rectum.
Echinacea-Colitis (Group 3; $\mathrm{n}=5$ ) were allowed ad libitum access to food, water and $50 \mathrm{mg} / \mathrm{kg}$ /day Echinacea for 14 days. On day eight, $1 \mathrm{ml}$ saline and $1 \mathrm{ml} \mathrm{4 \%}$ acetic acid was administered into the rectum.

Colitis (Group 4; $\mathrm{n}=5$ ) were allowed ad libitum access to food, water for 14 days. On day eight, $1 \mathrm{ml}$ saline and $1 \mathrm{ml} \mathrm{4 \%}$ acetic acid was administered into the rectum.

\section{Assessment of colonic damage}

All rats were euthanized by cervical decapitation under general anesthesia on day fifteen. The abdomen was opened and the colon was exposed. The distal $8 \mathrm{~cm}$ of the colon was excised and opened by longitudinal incision. After the colon segments had been scored macroscopically, the distal $4 \mathrm{~cm}$ of colon was preserved with $10 \%$ formalin for microscopic evaluation. The proximal $4 \mathrm{~cm}$ of colon segments were used for biochemical analysis (MDA, TAS) (Tabs 1 and 2). After washing the mucosa with saline solution, mucosal injury (macroscopically) was assessed using the grading scales as follows: score 0 : no damage, score 1: localized hyperemia but no ulcers, score 2: linear ulcers with no significant inflammation, score 3: linear ulcer with inflammation at one site, score 4: two or more sites of ulceration and inflammation, score 5: two or more sites of ulceration and inflammation or one major site of inflammation and ulceration extending $>1 \mathrm{~cm}$ along the length of the colon (15). Additional samples were preserved in $10 \%$ formalin for histological examination. Formalin-fixed colonic samples were embedded in paraffin, and sections were stained with hematoxylin and eosin.

\section{Biochemical analysis}

Malondialdehyde (MDA) levels were assayed as umol/gr with a commercial kit (OxisResearch, CA, USA) based on the colorimetric method using Shimadzu UV 1601 spectrophotometer (Shimadzu Corporation, Kyoto, Japan). MDA method is based on the reaction of a chromogenic reagent $\mathrm{N}$-methyl-2 phenylindole (NMPI) with MDA at $45^{\circ} \mathrm{C}$. One molecule of MDA reacts with 2 molecules of NMPI to yield a stable carbocyanine dye with maximum absorption at $586 \mathrm{~nm}$. The assay with intra- and interassay coefficients of variable was less than $5.0 \%$.

TAS (Total Antioxidative Status) levels were assayed as umol/ gr with commercial kit (Immundiagnostik, Bensheim, Germany) which is based on the colorimetric method using Shimadzu UV

Tab. 1. Effects of Echinacea spp. on macroscopy and microscopy.

\begin{tabular}{lcccc}
\hline & $\begin{array}{c}\text { Control (Group 1) } \\
(\mathrm{n}=5)\end{array}$ & $\begin{array}{c}\text { Echinacea (Group 2) } \\
(\mathrm{n}=5)\end{array}$ & $\begin{array}{c}\text { Echinacea-Colitis (Group 3) } \\
(\mathrm{n}=5)\end{array}$ & $\begin{array}{c}\text { Colitis (Group 4) } \\
(\mathrm{n}=5)\end{array}$ \\
\hline Macroscopy & $0 \pm 0$ & $0 \pm 0$ & $0.9 \pm 0.43$ & $4.1 \pm 0.2$ \\
Microscopy & $0.5 \pm 0.21$ & $0.5 \pm 0.25$ & $1.9 \pm 0.7$ & $<0.001$ \\
\hline
\end{tabular}

Values expressed as mean \pm SE. P* value: Colitis group campared with other groups. $\mathrm{P}^{* *}$ value: Groups of control, echinacea, echinacea-colitis compared with each other.

Tab. 2. Effects of Echinacea spp. on TAS and MDA.

\begin{tabular}{lcccc}
\hline & $\begin{array}{c}\text { Control (Group 1) } \\
(\mathrm{n}=5)\end{array}$ & $\begin{array}{c}\text { Echinacea (Group 2) } \\
(\mathrm{n}=5)\end{array}$ & $\begin{array}{c}\text { Echinacea-Colitis (Group 3) } \\
(\mathrm{n}=5)\end{array}$ & $\begin{array}{c}\text { Colitis (Group 4) } \\
(\mathrm{n}=5)\end{array}$ \\
\hline MDA & $0.36 \pm 0.05$ & $0.30 \pm 0.06$ & $0.39 \pm 0.14$ & $0.70 \pm 0.20$ \\
TAS & $2.87 \pm 0.75$ & $24.50 \pm 20.03$ & $1.96 \pm 0.58$ & $\mathrm{p}<$ \\
\hline
\end{tabular}

MDA: Malondialdehyde. TAS: Total antioxidant status. MDA and TAS were assigned as umol/gr. Values expressed as mean \pm SD. P* value: Group campared with other groups. $\mathrm{P} * *$ value: Group compared with colitis group. 


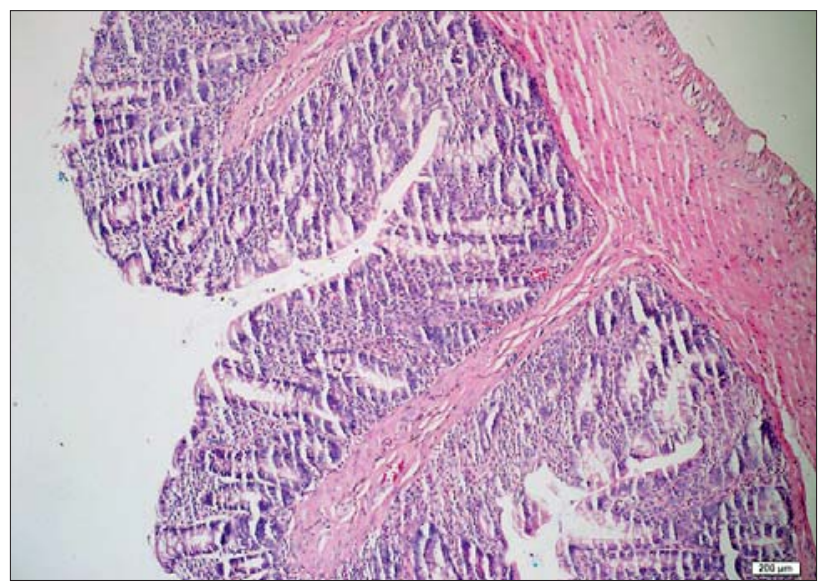

Fig. 1. Normal colonic tissue architecture of colon in control group.

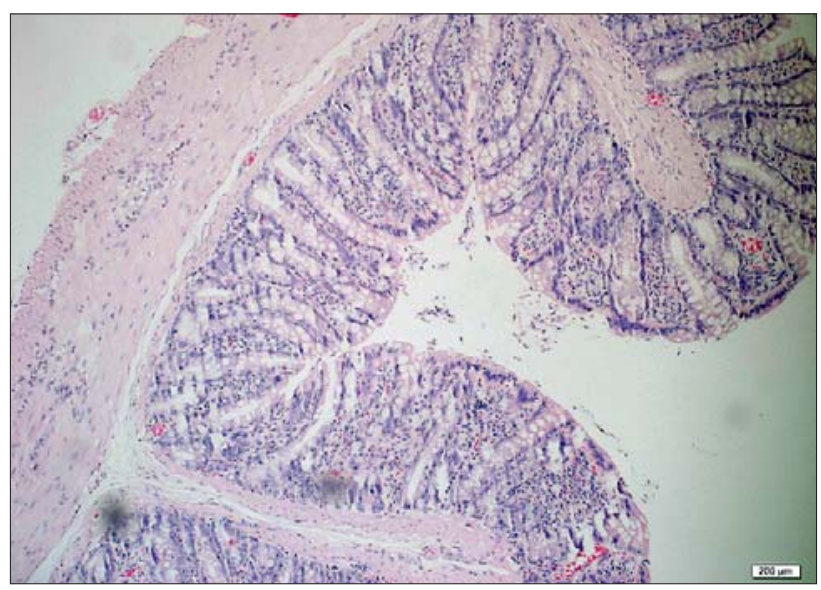

Fig. 2. Normal colonic tissue architecture of colon in echinaceal group.

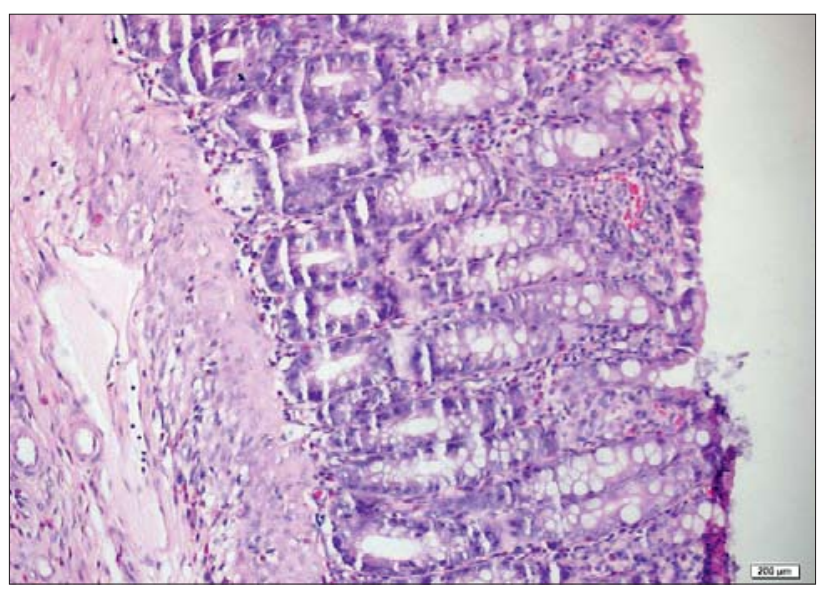

Fig. 3. Minimal changes in tissue architecture and cell infiltration in echinacea-colitis group.

1601 spectrophotometer (Shimadzu Corporation, Kyoto, Japan). The determination of antioxidative capacity is performed by the reaction of antioxidants in the sample with a defined amount of

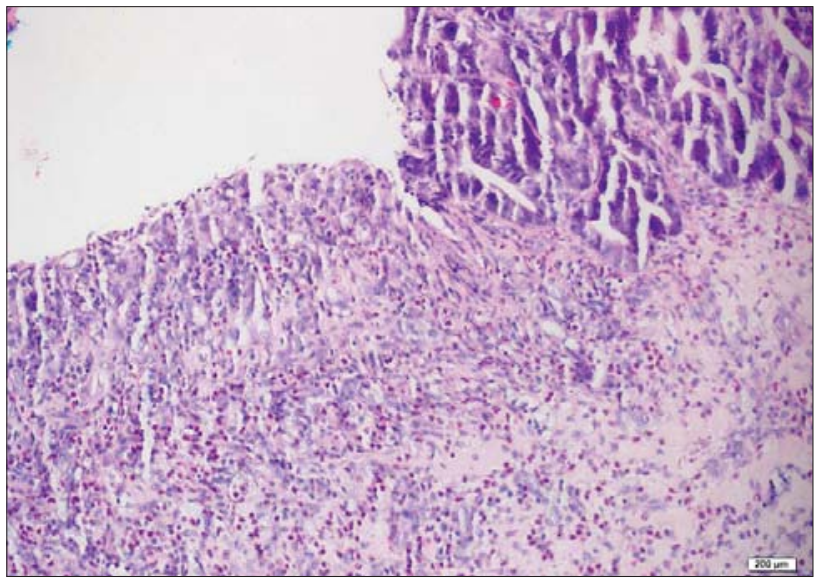

Fig. 4. Loss of epithelial cells with ulceration, goblet cell depletion with inflammatory cells infiltration in colitis group.

exogenously provided hydrogen peroxide $\left(\mathrm{H}_{2} \mathrm{O}_{2}\right)$. The residual $\mathrm{H}_{2} \mathrm{O}_{2}$ is determined photometrically by an ezymatic reaction which involves the conversion of TMB to a colored product. The assay with intra- and interassay coefficients of variable was less than $5.0 \%$.

\section{Statistical analysis}

Data are expressed as mean \pm SD. Statistical analyses were performed by one-way ANOVA followed by Tukey's multiple comparison test for levels of MDA and TAS. Kruskal-Wallis test was used for microscopic and macroscopic scoring and disease activity index with the Statistical Package for Social Sciences version 16.0 (SPSS Inc., Chicago, USA). A p value of less than 0.05 was considered statistically significant.

\section{Results}

In colitis group (Group 4), compared to other groups, the expected macroscopic features of ulcerative colitis e.g. multiple ulcers and diffuse inflammation were noticed macroscopically. The histopathologic examination revealed mucosal inflammation. Multiple ulcerations were also noticed, indicating the presence of a crypt abscess. Glandular destruction and goblet cell depletion were also seen. In the submucosa, multifocal areas of inflammation and ulceration were present and it was diffusely edematous. Infiltration of polymorphonuclear leukocytes, eosinophils and lymphocytes was extensive ( $x 400, \mathrm{H}$ and E) (Fig. 4) $(\mathrm{p}<0.001)$. In contrast, macroscopic and microscopic evaluation showed mild changes in control, Echinacea and Echinacea-colitis groups (Figs $1,2,3)(\mathrm{p}>0.3 ; \mathrm{p}>0.21$, respectively $)$

MDA levels were significantly lower in Echinacea group (Group 2) $(\mathrm{p}<0.001)$. Similarly, MDA levels were also lower in Echinacea-colitis group (Group 3) compared to colitis group $(\mathrm{p}<0.001)$.

TAS was found to be higher in Echinacea group compared to other groups $(p<0.001)$. TAS was also higher in Echinacea-colitis group compared to colitis group $(\mathrm{p}<0.001)$. 


\section{Discussion}

In this study, we evaluated the antioxidant effects of Echinacea spp. in rat colitis model induced by acetic acid. Upon macroscopic and microscopic examinations of colon, we observed a protective effect of Echinacea spp. administration. The changes in antioxidant cytokines MDA and TAS support these histological changes. The cause of this effect is likely due to antioxidants in E. purpurea, such as echinacoside and caffeic acid (20). Echinacea is an antioxidant agent effective in eliminating free radicals produced by irradiation with its radical-scavenging effect, reducing cytotoxicity caused by lipid peroxides due to oxidization, and preventing impairment of immunity in mice exposed to radiation (21).

Regarding UC induction, there are several reports on different chemicals to induce experimental colitis, such as acetic acid, 2,4,6-trinitrobenzene sulfonic acid, dextran sodium sulfate, oxazolone and indomethacin (22-26). In this study, $4 \%$ acetic acid was used to induce UC. Acetic acid is an easily available inexpensive chemical agent. Our findings confirmed the induction of UC both macroscopically and histologically. Acetic acid causes infiltration of colonic mucosa by neutrophils and macrophages and increases production of inflammatory mediators in experimental colitis model. The mechanism resulting in formation of the lesion is thought to be an acute inflammatory response following colonic injury caused by nonspecific acids accompanied with widespread hemorrhage, and release of mediators (27).

Although the precise mechanism of UC still remains obscure, the role of reactive oxidative metabolites is becoming increasingly evident. The increase in reactive oxidative metabolites within colonic tissues plays a pivotal role in the pathogenesis of UC (28). Serum MDA levels are significantly increased in UC rats in correlation with the severity of disease in previous studies. It indicates that ROMs are closely involved in the inflammatory responses in $\mathrm{UC}$ (29). Oxidative stress is a valid etiological or triggering factor for UC, because the harmfull effects of ROMs have been shown in the inflammation process (30). In several studies, the level of ROMs was found to increase in animal colitis models induced by different molecules such as acetic acid and dextran sodium sulfate $(31,32)$. Interestingly, MDA and TAS levels differed between Group 3 (Echinacea-colitis group) and colitis group in colonic tissue ( $p<0.001 ; p<0,001$, respectively). Significantly higher TAS levels, lower MDA levels, and mild histological changes in Group 3 (Echinacea-colitis group) compared to Group 4 (colitis group) show the protective role of Echinacea in relation to colitis $(\mathrm{p}<0.001 ; \mathrm{p}<0.001 ; \mathrm{p}<0.001$, respectively)

The level of MDA was significantly lower and the level of TAS was significantly higher in Group 2 (Echinacea group) compared to control group, in our study meaning that Echinacea has no harmful effect on normal colon ( $\mathrm{p}<0,001 ; \mathrm{p}<0,001$, respectively).

There are some limitations to this study. Firstly, histopathological features were defined as microscopic and macroscopic features but with no histological score comparison. The other limitation is lack of other assays for oxidative stress, such as SOD and MPO.

Previous studies showed to decrease the severity and reduce the duration of colds and upper respiratory infections, especially when Echinacea is taken early in the course of infection $(33,34)$. A recent case-control study showed the favourable effect of Echinacea on gastrointestinal mucosal recovery in children with mucositis due to chemotherapy (35). Protection of colonic mucosa is probably related, at least in part, to the antioxidative properties of Echinacea. If the present findings can be supported in clinical studies, Echinacea will possibly have some therapeutic usefulness in the management of human UC. It could be used as an add-on to standard medication to help reach and maintain remission.

\section{References}

1. Fiocchi C. Inflammatory bowel disease: etiology and pathogenesis. Gastroenterology 1998; 115: 182-205.

2. Papadakis KA, Targan SR. Role of cytokines in the pathogenesis of inflammatory bowel disease. Ann Rev Med 2000; 51: 289-298.

3. Rogler G, Andus T. Cytokines in inflammatory bowel disease. World J Surg 1998; 22: 382-389.

4. Ogata H, Hibi T. Cytokine and anti-cytokine therapies for inflammatory bowel disease. Curr Pharm Design 2003; 14: 1107-1113.

5. Weiss SJ. Tissue destruction by neutrophils. N Engl J Med 1989; 320 : 365-379.

6. Cross CE, Halliwell B, Borish ET et al. Oxygen radicals and human disease. Ann Intern Med 1987; 107: 526-545.

7. Parks P, Ruckley G, Granger N. Role of oxygen-derived free radicals in digestive tract disease. Surgery 1983; 94: 415-422.

8. Bertoglio JC, Folatre I, Bombardelli E, Riva A, Morazzoni P, Ronchi M, Petrangolini G. Management of gastrointestinal mucositis due to cancer therapies in pediatric patients: results of a case series with SAMITAL. Future Oncol 2012; 8: 1481-1486.

9. Bauer R. New knowledge regarding the effect and effectiveness of Echinacea purpurea extracts. Wien Med Wochenschr. 2002; 152: 407-411.

10. Goel C, Chang C, Slama JV, Barton R, Bauer R, Gahler R, Basu TK. Int Immunopharmacol 2002; 381-387.

11. Weiss SJ. Oxygen, ischemia and inflammation. Acm Physiol Scnnd 1986; 548 (Suppl): Y-37.

12. Hruszkewycz AM. Lipid peroxidation and mt DNA degeneration. A hypothesis. Mutar Kes 1992; 275: 243-248.

13. Yao J, Wang JY, Liu L, Li YX, Xun AY, Zeng WS, Jia CH, Wei XX, Feng JL, Zhao L, Wang LS. Anti-oxidant effects of resveratrol on mice with DSS- induced ulcerative colitis. Arch Med Res 2010; 41: 288-294.

14. Amirshahrokhi K, Bohlooli S, Chininifroush MM. The effect of methylsulfonylmethane on the experimental colitis in the rat. Toxicol Appl Pharmacol 2011; 253: 197-202.

15. Pavlick KP, Laroux FS, Fuseler $\mathbf{J}$ et al. Role of reactive metabolites of oxygen and nitrogen in inflammatory bowel disease. Free Radic Biol Med 2002; 33: 311-322.

16. Keshavarzian A, Morgan G, Sedghi S, Gordon JH and Doria M. Role of reactive oxygen metabolites in experimental colitis. Gut 1990; 31: 786-790.

17. Millar A D, Rampton DS, Chander CL, Claxson AW, Blades S, Coumbe A et al. Evaluating the antioxidant potential of new treatments for inflammatory bowel disease using a rat model of colitis. Gut 1996; 39: $407-415$. 
18. Ferkolj I. How to improve the safety of biologic therapy in Crohn's disease. J Physiol Pharmacol 2009; 60: 67-70.

19. Ha C, Dassopoulos T. Thiopurine therapy in inflammatory bowel disease. Expert Rev Gastroenterol Hepatol 2010; 4: 575-588.

20. Sloley BD, Urichuk LJ, Tywin C, Coutts RT, Pang PK, Shan JJ. J Pharm Pharmacol 2001; 53: 849-857.

21. Mishima S, Saito K, Maruyama H, Inoue M, Yamashita T, Ishida T, Gu Y. Antioxidant and immuno-enhancing effects of Echinacea purpurea. Biol. Pharm. Bull2004; 27: 1004-1009.

22. Bauer C, Duewell P, Lehr HA, Endres S, Schnurr M. Protective and aggravating effects of NIrp3 inflammasome activation in IBD models: influence of genetic and environmental factors. Dig Dis 2012; 30: 82-90.

23. Strober W, Fuss IJ, Blumberg RS. The immunology of mocosal models of inflammation. Annu Rev Immunol 2002; 20: 495-549.

24. Kawada M, Arihiro A, Mizoguchi E. İnsights from advances in research of chemically induced experimental models of human inflammatory bowel disease. World J Gastroenterol 2007; 13: 5581-5593.

25. Heller F, Fuss IJ, Nieuwenhuis EE, Blumberg RS, Strober W. Oxazolone colitis, a Th2 colitis model resembling ulcerative colitis, is mediated by IL-13-producing NK-T cells. Immunity 2002; 17: 629-638.

26. Standnyk AW, Dollard C, Issekutz TB, Issekutz AC. Neutrophil migration into indomethacin induced rat small intestinal injury is CD11a/ CD18 and CD11b/CD18 co-dependent. Gut 2002; 50: 629-635.

27. Fabia R, Willen R, ArRajab A, Andersson R, Ahren B, Bengmark $\mathrm{S}$. Acetic acid-induced colitis in the rat: a reproducible experimental model for acute ulcerative colitis. Eur Surg Res 1992; 24: 211-225.
28. Andoh A, Yagi Y, Shioya M, Nishida A, Tsujikawa T, Fujiyama Y. Mucosal cytokine network in inflammatory bowel disease. World J Gastroenterol 2008; 33: 5154-5161.

29. Autenrieth I, Bucheler N, Bohn E, Heinze G, Horak I. Cytokine mRNA expression in intestinal tissue of interleukin-2 deficient mice with bowel inflammation. 1997; 41: 793-800.

30. Spitz DR et al. Metabolic oxidation/reduction reactions and cellular responses to ionizing radiation: a unifying concept in stress response biology. Cancer Metastasis Rev 2004; 23: 311-322.

31. Ghafari GMB, Yasa N, Ashtaral-Nakhai L, Mohammadirad A, Nikfar S, Dehghan G, Shetab-Boushehri V, Jamshidi H, Khorassani R, Salehnia A, Abdollahi M. Biochemical and histopathological evidences for beneficial effects of Satureja khuzestanica jamzad essential oil on the Mouse model of inflammatory bowel diseases. Toxicology Mechanisms Methods 2006; 16: 365-372.

32. Tham DM, Whitin JC, Cohen HJ. Increased expression of extracellular glutathione peroxidase in mice with dextran sodium sulfate-induced experimental colitis. Pediat Res 2002; 51: 641-646.

33. Linde K, Barrett B, Wölkart K, Bauer R, Melchart D. Echinacea for preventing and treating the common cold.

34. Thomas P. Echinacea: a natural remedy for the common cold and flu? Nutr Today 2001; 36: 249-253.

35. Bertoglio JC, Folatre I, Bombardelli E, Riva A, Morazzoni P, Ronchi M, Petrangolini G. Management of gastrointestinal mucositis due to cancer therapies in pediatric patients: results of a case series with SAMITAL. Future Oncol 2012; 8: 1481-1486. 